RESEARCH ArTICLE

Published December 3, 2021

\title{
SOluble Tumor Necrosis FACTOR RECEPTOR 1 IS ASSOCIATED WITH Cardiovascular Risk in Persons With Coronary Artery Calcium SCORE OF ZERO
}

\section{AUTHORS}

Tony Dong, $\mathrm{MD}^{1}$; Graham Bevan, $\mathrm{MD}^{1}$; David A Zidar, $\mathrm{MD}, \mathrm{PhD}^{1,2,3,4}$; Miguel Cainzos Achirica, MD, $\mathrm{MPH}^{5}$; Khurram Nasir, MD, MS 5 ; Imran Rashid, MD, PhD ${ }^{1,2,4}$; Sanjay Rajagopalan, $\mathrm{MD}^{1,2,4}$; Sadeer Al-Kindi, $\mathrm{MD}^{1,2,4}$

\section{AFFILIATED INSTITUTIONS}

${ }^{1}$ Department of Medicine, University Hospitals, Cleveland, Ohio ${ }^{2}$ Louis Stokes Cleveland Veterans Affairs Hospital, Cleveland, Ohio ${ }^{3}$ School of Medicine, Case Western Reserve University, Cleveland, Ohio ${ }^{4}$ Harrington Heart and Vascular Institute, University Hospitals, Cleveland, Ohio ${ }^{5}$ Houston Methodist Hospital, Houston, Texas

CORRESPONDING AUTHOR

Sadeer Al-Kindi, MD, FACC

Assistant Professor of Medicine and Radiology

University Hospitals Harrington Heart and Vascular Institute

Case Western Reserve University School of Medicine

11100 Euclid Ave, Cleveland, OH 44106

Email: sadeer.alkindi@uhhospital.org

Phone: 216-844-1639, Fax: 216-844-8954
DOI

10.20411/pai.v6i2.477

\section{SUGGESTED CITATION}

Dong T, Bevan G, Zidar DA, Achirica MC, Nasir K, Rashid I, Rajagopalan S, Al-Kindi S. Soluble Tumor Necrosis Factor Receptor 1 is Associated with Cardiovascular Risk in Persons with Coronary Artery Calcium Score of Zero. Pathogens and Immunity. 2021;6(2): 135-148. doi: 10.20411/ pai.v6i2.477 


\section{ABSTRACT}

Background: A coronary artery calcium (CAC) score of zero confers a low but nonzero risk of atherosclerotic cardiovascular events (CVD) in asymptomatic patient populations, and additional risk stratification is needed to guide preventive interventions. Soluble tumor necrosis factor receptors (sTNFR-1 and sTNFR-2) are shed in the context of TNF-alpha signaling and systemic inflammation, which play a role in atherosclerosis and plaque instability. We hypothesized that serum sTNFR-1 concentrations may aid in cardiovascular risk stratification among asymptomatic patients with a CAC score of zero.

Methods: We included all participants with $\mathrm{CAC}=0$ and baseline sTNFR- 1 measurements from the prospective cohort Multi-Ethnic Study of Atherosclerosis (MESA). The primary outcome was a composite CVD event (myocardial infarction, stroke, coronary revascularization, cardiovascular death).

Results: The study included 1471 participants (mean age 57.6 years, 64\% female), with measured baseline sTNFR-1 ranging from $603 \mathrm{pg} / \mathrm{mL}$ to $5544 \mathrm{pg} / \mathrm{mL}$ (mean $1294 \mathrm{pg} / \mathrm{mL} \pm 378.8 \mathrm{pg} / \mathrm{mL}$ ). Over a median follow-up of 8.5 years, 37 participants (2.5\%) experienced a CVD event. In multivariable analyses adjusted for Framingham Score, doubling of sTNFR-1 was associated with a 3 -fold increase in the hazards of CVD (HR 3.0, 95\% CI: 1.48- 6.09, $P=0.002$ ), which remained significant after adjusting for traditional CVD risk factors individually (HR 2.29; $95 \%$ CI: $1.04-$ $5.06, P=0.04)$. Doubling of sTNFR-1 was also associated with progression of CAC $>100$, adjusted for age (OR 2.84, 95\% CI: 1.33-6.03, $P=0.007$ ).

Conclusions: sTNFR- 1 concentrations are associated with more CVD events in participants with a CAC score of zero. Utilizing sTNFR-1 measurements may improve cardiovascular risk stratification and guide primary prevention in otherwise low-risk individuals.

\section{KEYWORDS}

soluble tumor necrosis factor receptor; TNF alpha; coronary calcium score; cardiovascular risk stratification; MESA

\section{INTRODUCTION}

The pathogenesis of coronary artery disease (CAD) is complex and is characterized by abnormal lipid metabolism and chronic inflammation, leading to endothelial dysfunction and subintimal deposition of low-density lipoproteins (LDL) [1]. The importance of pro-inflammatory pathways in accelerating atherosclerosis is extensively demonstrated in experimental studies and translates directly to clinical utility [2] . C-reactive protein (CRP), a downstream inflammatory biomarker, has been linked to increased cardiovascular risk independent of traditional risk factors [ $\underline{3}$ ] and is included in some risk scores for cardiovascular risk stratification [4]. Lipid-lowering therapies are considered to impart anti-inflammatory effects, and they were shown to decrease cardiovascular events in patients with residual inflammatory risk [ [5]. Recently, the concept of causality between inflammation and atherosclerosis has been proposed with the CANTOS trial, which showed that anti-inflammatory therapy with canakinumab resulted in a significant reduction in adverse cardiovascular events [6]. Soluble tumor necrosis factor receptors (sTNFR-1 and sTNFR-2) are shed during TNF- $\alpha$ cytokine signaling. Circulating levels of sTNFR- 1 and sTNFR-2 have been shown to increase with TNF- $\alpha$ activity in response to pro-inflammatory stimuli []]. They are useful markers of TNF- $\alpha$ activity due to their long half-lives in the serum. TNF- $\alpha$ has been linked to 
increased cardiovascular events in a 5-year period post myocardial infarction [ $\underline{8}$ ] as well as CAD complexity [9]. Several TNF-alpha blockers are approved for use in patients with chronic inflammatory conditions, such as rheumatoid arthritis and inflammatory bowel disease.

Coronary artery calcium (CAC) scoring has become increasingly utilized for cardiovascular risk stratification and is endorsed by the guidelines in intermediate-risk patients. The degree of vascular intimal calcification is directly related to progression of atherosclerosis, and extensive evidence has shown that CAC of zero is associated with very low risk of CVD risk in asymptomatic populations [10-12]. However, a CAC score does not account for noncalcified coronary plaques, which are often less stable and more prone to plaque rupture [13]. Noncalcified coronary plaques are more prevalent in patients with inflammatory diseases (eg, rheumatoid arthritis, HIV, lupus)[14], suggesting a possible correlation with increased systemic inflammation. Thus, it is possible that inflammatory biomarkers increase CVD risk even in patients with zero coronary artery calcium. Here, we investigated the role of serum sTNFR-1 as an inflammatory biomarker in cardiovascular risk stratification for asymptomatic persons with a CAC score of zero.

\section{METHODS}

\section{Population}

Data was obtained from the Multi-Ethnic Study of Atherosclerosis (MESA). The MESA is a prospective cohort study that enrolled 6814 asymptomatic adult participants aged 45 to 84 years between July 2000 and August 2002 from 6 centers in the United States [15]. Baseline characteristics were obtained including demographics, physical exams, cardiovascular risk factors, and CAC score [16]. The CAC scores were obtained using electron-beam and multi-detector row CT. All patients were scanned twice at the first visit and at 5 subsequent follow-up visits, and the mean CAC Agatston score for each visit was used for our analysis. As part of the MESA Family ancillary study, serum sTNFR-1 concentrations were also measured at baseline in a subgroup of 2871 race/ ethnicity matched patients. Serum sTNFR-1 concentrations were measured using ultrasensitive ELISA (R\&D Systems, Minneapolis, Minnesota) at a central laboratory (University of Vermont, Burlington, Vermont). The MESA Family ancillary study selected an ethnically diverse population for genetic studies and included an even number of patients from each ethnicity (White, African American, Asian, and Hispanic), resulting in a larger proportion of Asians (23.7\%) compared to the original MESA cohort (11.6\%). We also explored additional inflammatory markers (C-reactive protein [CRP] and Interleukin-6 [IL-6]) for comparative purposes. Serum CRP levels were measured using the BNII nephelometer (N High Sensitivity CRP, Dade Behring, Deerfield, IL; CV $2.1 \%-5.7 \%$ ); the lower limit of detection was $0.16 \mathrm{mg} / \mathrm{mL}$. IL-6 was measured by ultra-sensitive sandwich ELISA (Quantikine HS Human IL-6 Immunoassay; R\&D Systems, Minneapolis, Minnesota; CV 6.3\%). For our study, we included all participants with a CAC score of zero and with measured baseline serum sTNFR-1 concentrations at initial study enrollment. A total of 1471 patients met these criteria. As a part of the MESA study, these participants were followed up over a 10-year period for all CVD events. CVD events were defined as a composite of myocardial infarction (MI), resuscitated cardiac arrest, definite angina, probable angina if followed by revascularization, stroke, and CVD death. Hard CVD events were defined as a composite of myocardial infarction, resuscitated cardiac arrest, cardiovascular disease-related death, stroke, or stroke-related death. All events were adjudicated by a central committee as previously described [17]. 


\section{Statistical Analysis}

All patients were initially stratified into baseline $\mathrm{CAC}=0$ and $\mathrm{CAC}>0$, and mean sTNFR-1 measurements were compared using analysis of variance (ANOVA). Then, baseline characteristics for patients with $\mathrm{CAC}=0$ were calculated including demographics (age, sex, and race), baseline lipid panel (low density lipoprotein (LDL), high density lipoprotein (HDL), triglycerides (TG) and total cholesterol (TC)), and traditional cardiovascular risk factors (family history of MI, history of hypertension, obesity, diabetes, and smoking). Race was divided into categories of White, African American, Asian, and Hispanic. Obesity was divided into body mass index (BMI) categories of $<25,25-30,30-40$, and $>40 \mathrm{~kg} / \mathrm{m}^{2}$. Diabetes was divided into categories of no diabetes, pre-diabetes (defined as fasting glucose $\geq 100 \mathrm{mg} / \mathrm{dL}$ ), untreated diabetes, and treated diabetes based on a fasting glucose cutoff $\geq 126 \mathrm{mg} / \mathrm{dL}$. Smoking history was a binary variable (current smoker and non-current smoker). Patients were then divided into sTNFR-1 tertiles, and baseline characteristics were calculated for each subgroup. Baseline characteristics were compared between each subgroup using ANOVA for continuous variables and chi-square analysis for categorical variables.

Kaplan-Meier curves were constructed for all participants using time to all CVD events, with censoring of participants who left the study prior to completion. Kaplan-Meier curves were also constructed for each sTNFR-1 tertile subgroup. To measure the association between sTNFR-1 and all CVD events, a multivariate Cox proportional-hazards regression model was implemented using the binary logarithm of sTNFR-1 ( $\log _{2}$ sTNFR-1) as a continuous variable and including traditional cardiovascular risk factors (age, sex, race, diabetes, smoking, total cholesterol, HDL cholesterol, systolic blood pressure, diastolic blood pressure, and antihypertensive medication use). This was repeated for hard CVD events (composite of MI, death from MI, resuscitated cardiac arrest, stroke, and death from stroke), MI alone, stroke alone, and deaths alone. Subgroup analysis was performed on participants stratified by age ( $\geq 55$ years, $<55$ years), sex, and Risk Score $(\geq 7.5,<7.5)$. The predicted 10 -year risk of all CVD events with respect to serum sTNFR-1 concentration was calculated using a Cox proportional-hazards regression model adjusting for Framingham Risk Score. The C-statistic was calculated using the area under the curve (AUC) of the receiver operating characteristics (ROC) for the Framingham Risk Score alone, and the Cox proportional-hazards regression model for Framingham Risk Score and sTNFR-1. ROCs were also calculated for 2 other inflammatory markers: interleukin- 6 and CRP. The DeLong test was used to compare AUCs. All survival analyses were performed in R version 4.0.4 using the packages survival and rms. All AUC calculations were performed using the package pROC. The distribution of calcium score progression in 4 subsequent visits, stratified by sTNFR-1 tertile was compared using chi-square analysis.

\section{RESULTS}

A total of 6814 participants were included in the MESA. There were 3390 participants (49.8\%) who had a baseline CAC of zero, and 1471 participants (mean age $57.6 \pm 9.1,35.9 \%$ men) had available sTNFR-1 measurements and were included in our study. sTNFR-1 measurements ranged from $603 \mathrm{pg} / \mathrm{mL}$ to $5544 \mathrm{pg} / \mathrm{mL}$ (mean $1294 \mathrm{pg} / \mathrm{mL} \pm 378.8 \mathrm{pg} / \mathrm{mL}$ ), which was lower than participants with a baseline CAC $>0(\mathrm{n}=1400$, mean $1466 \mathrm{pg} / \mathrm{mL} \pm 468.5 \mathrm{pg} / \mathrm{mL}, P<0.001)$. The baseline characteristics at initial enrollment are reported for the entire population and by sTNFR-1 tertiles (Table 1). sTNFR-1 was associated with older age, male sex, White race, obesity, diabetes, and smoking. 
Table 1. Participant characteristics by sTNFR-1 tertiles

\begin{tabular}{|c|c|c|c|c|c|}
\hline $\begin{array}{l}\text { Participant } \\
\text { Characteristics }\end{array}$ & All & 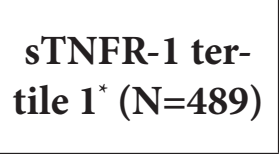 & $\begin{array}{l}\text { sTNFR-1 } \\
\text { tertile } 2^{*} \\
(\mathrm{~N}=490)\end{array}$ & $\begin{array}{l}\text { sTNFR-1 } \\
\text { tertile } 3^{*} \\
(\mathrm{~N}=492)\end{array}$ & $P$ value \\
\hline Age (years, SD) & $57.6(9.1)$ & $54.8(7.6)$ & $57.1(8.5)$ & $61.0(10.0)$ & $<0.001$ \\
\hline $\begin{array}{l}\text { Sex } \\
\text { Male } \\
\text { Female }\end{array}$ & $\begin{array}{l}528(35.9 \%) \\
943(64.1 \%)\end{array}$ & $\begin{array}{l}149(30.3 \%) \\
343(69.7 \%)\end{array}$ & $\begin{array}{l}191(39 \%) \\
299(61 \%)\end{array}$ & $\begin{array}{l}188(38.4 \%) \\
301(61.6 \%)\end{array}$ & 0.006 \\
\hline $\begin{array}{l}\text { Race } \\
\text { White } \\
\text { African American } \\
\text { Asian } \\
\text { Hispanic }\end{array}$ & $\begin{array}{l}322(21.9 \%) \\
407(27.7 \%) \\
349(23.7 \%) \\
393(26.7 \%)\end{array}$ & $\begin{array}{l}90(18.3 \%) \\
146(29.7 \%) \\
155(31.5 \%) \\
101(20.5 \%)\end{array}$ & $\begin{array}{l}110(22.4 \%) \\
137(28 \%) \\
110(22.4 \%) \\
133(27.1 \%)\end{array}$ & $\begin{array}{c}122(24.9 \%) \\
124(25.4 \%) \\
84(17.2 \%) \\
159(32.5 \%)\end{array}$ & $<0.001$ \\
\hline Hypertension & $958(65.1 \%)$ & $359(73 \%)$ & $334(68.2 \%)$ & $265(54.2 \%)$ & $<0.001$ \\
\hline $\begin{array}{l}\text { Obesity } \\
\qquad \text { BMI }<25 \mathrm{~kg} / \mathrm{m} 2 \\
\text { BMI } 25-30 \mathrm{~kg} / \mathrm{m} 2 \\
\text { BMI } 30-40 \mathrm{~kg} / \mathrm{m} 2 \\
\text { BMI }>40 \mathrm{~kg} / \mathrm{m} 2\end{array}$ & $\begin{array}{c}475(32.3 \%) \\
560(38.1 \%) \\
380(25.8 \%) \\
56(3.8 \%)\end{array}$ & $\begin{array}{c}221(44.9 \%) \\
182(37 \%) \\
83(16.9 \%) \\
6(1.2 \%)\end{array}$ & $\begin{array}{c}155(31.6 \%) \\
184(37.6 \%) \\
138(28.2 \%) \\
13(2.7 \%)\end{array}$ & $\begin{array}{c}99(20.2 \%) \\
194(39.7 \%) \\
159(32.5 \%) \\
37(7.6 \%)\end{array}$ & $<0.001$ \\
\hline $\begin{array}{l}\text { Diabetes mellitus, type II } \\
\text { None } \\
\text { Insulin Resistance } \\
\text { Diabetes, untreated } \\
\text { Diabetes, on treatment }\end{array}$ & $\begin{array}{c}1139(77.6 \%) \\
195(13.3 \%) \\
31(2.1 \%) \\
103(7 \%)\end{array}$ & $\begin{array}{c}400(81.5 \%) \\
53(10.8 \%) \\
15(3.1 \%) \\
23(4.7 \%)\end{array}$ & $\begin{array}{c}397(81 \%) \\
60(12.2 \%) \\
7(1.4 \%) \\
26(5.3 \%)\end{array}$ & $\begin{array}{c}342(70.2 \%) \\
82(16.8 \%) \\
9(1.8 \%) \\
54(11.1 \%)\end{array}$ & $<0.001$ \\
\hline Current Smoker & $201(13.7 \%)$ & $52(10.6 \%)$ & $73(14.9 \%)$ & $76(15.5 \%)$ & 0.048 \\
\hline Family history of MI & $469(33.8 \%)$ & $126(27 \%)$ & $161(34.8 \%)$ & $182(39.9 \%)$ & $<0.001$ \\
\hline $\begin{array}{l}\text { Lipid Profile }(\mathrm{mg} / \mathrm{dL}, \mathrm{SD}) \\
\text { LDL-C }(\mathrm{mg} / \mathrm{dL})^{1} \\
\text { HDL-C }(\mathrm{mg} / \mathrm{dL})^{2} \\
\text { TG }(\mathrm{mg} / \mathrm{dL})^{3} \\
\text { TC }(\mathrm{mg} / \mathrm{dL})^{4}\end{array}$ & $\begin{array}{l}115.5(30.0) \\
52.1(14.5) \\
124.6(65.2) \\
192.5(33.0)\end{array}$ & $\begin{array}{l}114.3(29.9) \\
54.9(15.5) \\
116.9(62.4) \\
192.6(32.3)\end{array}$ & $\begin{array}{l}119.6(29.7) \\
51.7(13.9) \\
122.2(62.0) \\
195.7(32.5)\end{array}$ & $\begin{array}{l}112.6(29.8) \\
49.65(13.7) \\
134.8(69.8) \\
189.2(33.9)\end{array}$ & $\begin{array}{c}0.381 \\
<0.001 \\
<0.001 \\
0.111\end{array}$ \\
\hline
\end{tabular}

${ }^{*}$ Tertile 1: 603 - $1118 \mathrm{pg} / \mathrm{mL}$, Tertile 2: 1119 - $1367 \mathrm{pg} / \mathrm{mL}$, Tertile 3: 1368 - 5544 pg/mL, ${ }^{1}$ Low-density lipoprotein cholesterol, ${ }^{2} \mathrm{High}$-density lipoprotein cholesterol, ${ }^{3}$ Triglycerides, ${ }^{4}$ Total Cholesterol

Over the total follow-up period of 10.9 years, 37 participants (2.5\%) experienced a CVD event, of which 31 were hard events (12 MI events, 14 stroke events, 1 stroke death, and 4 other cardiovascular deaths). There were also 46 non-cardiovascular deaths. The Kaplan-Meier curve for all CVD events is shown for the entire population and stratified by sTNFR-1 tertiles (Figure 1).

The binary logarithm of sTNFR-1 ( $\log _{2}$ sTNFR-1) was a significant predictor of all CVD events 
(HR $2.29[1.04,5.06], P=0.04)$ after accounting for traditional cardiovascular risk factors. Smoking was also a strong positive predictor (HR $3.65[1.69,7.90], P=0.001)$ and African American race was protective compared to White race (HR 0.36 [0.14, 0.92], $P=0.033$ ). In multivariable analysis adjusted for Framingham Score, doubling of sTNFR-1 was associated with 3-fold increase in risk of CVD (HR 3.0, 95\% [1.48, 6.09], $P=0.002$ ) (Table 2). The relationship of sTNFR-1 levels with modelled 10-year risk of all CVD (adjusted for Framingham Risk Score) is shown (Figure 2). For hard CVD events, $\log _{2}$ sTNFR-1 continued to be a strong predictor (HR $2.84[1.223,6.576]$, $P=0.015)$, along with smoking (HR $3.59[1.52,8.45], P=0.003)$.

For prediction of all CVD events, the AUC of the ROC of Framingham Risk Score alone was 0.721, compared to an AUC of 0.737 for a composite model of Framingham Risk Score with $\log _{2}$ sTNFR-1 $(P=0.009)$. In addition, sTNFR-1 tertile was associated with CAC progression at subsequent follow-up visits 3 and 5 ( $P<0.001$ and $P=0.002$, respectively), as shown (Figure 3 ). Doubling of sTNFR-1 was associated with progression of CAC>100 at visit 4 (OR 12.93 [2.24, 74.63], $P=0.004)$ and visit 5 (OR 2.84 [1.33, 6.03], $P=0.007$ ), after adjusting for age.
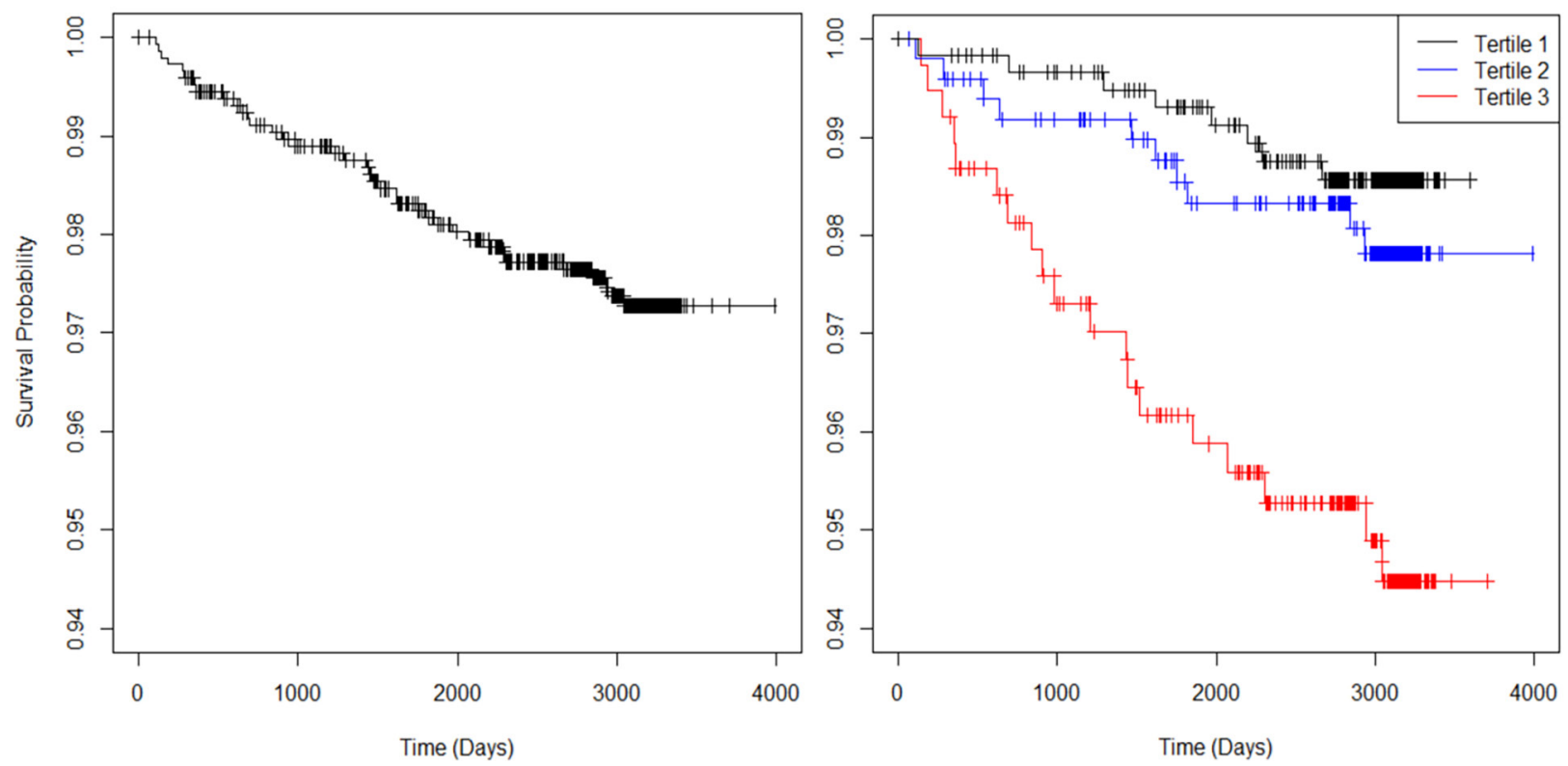

Figure 1. Kaplan-Meier curves with respect to all CVD events for all participants (left) and stratified by sTNFR-1 tertiles (right). 
Table 2. Cox proportional-hazards regression models for all CVD for the binary logarithm of sTNFR-1.

\begin{tabular}{|l|c|c|c|}
\cline { 2 - 4 } \multicolumn{1}{c|}{} & \multicolumn{3}{c|}{ All CVD (N=37) } \\
\cline { 2 - 4 } \multicolumn{1}{c|}{} & HR & 95\% CI & P \\
\hline $\log _{2}$ (sTNFR-1) adjusted for cardiovascular risk factors ${ }^{2}$ & 2.29 & $(1.04,5.06)$ & 0.040 \\
\hline $\log _{2}$ (sTNFR-1) adjusted for Framingham Risk Score & 3.00 & $(1.48,6.09)$ & 0.002 \\
\hline
\end{tabular}

${ }^{1}$ binary logarithm of sTNFR-1, ${ }^{2}$ Risk factors included: age, sex, race, diabetes, smoking, total cholesterol, HDL cholesterol, systolic blood pressure, diastolic blood pressure, and antihypertensive medication use.

When analyzed separately, $\log _{2}$ sTNFR-1 was a statistically significant predictor of stroke events (HR 3.59 [1.04, 12.44], $P=0.044$ ) and of all-cause mortality (HR 3.03 [1.53, 6.01], $P=0.002$ ), but did not reach statistical significance for MI events (HR $2.72[0.797,9.267], P=0.11$ ) after accounting for traditional cardiovascular risk factors.

In subgroup analysis by age, $\log _{2}$ sTNFR- 1 was a significant predictor of all CVD in participants under 55 years (HR 10.0 [1.57, 64.45], $P=0.015$ ) and 55 years or older (HR 2.68 [1.196, 6.021], $P=0.017)$. In subgroup analysis by sex and Framingham Risk Score, $\log _{2}$ sTNFR-1 was a significant predictor in male participants and a high Framingham Risk Score $(\geq 7.5)$, but not in female participants or a low Framingham Risk Score (<7.5) (Supplemental Table 1). In comparing sTNFR-1 to other available inflammatory markers for prediction of all CVD events, the AUC of the ROC of sTNFR-1 was 0.671, compared to an AUC of 0.570 for interleukin-6 $(P=0.06)$ and AUC of 0.503 for CRP $(P=0.002)$ (Supplemental Figure 1). 


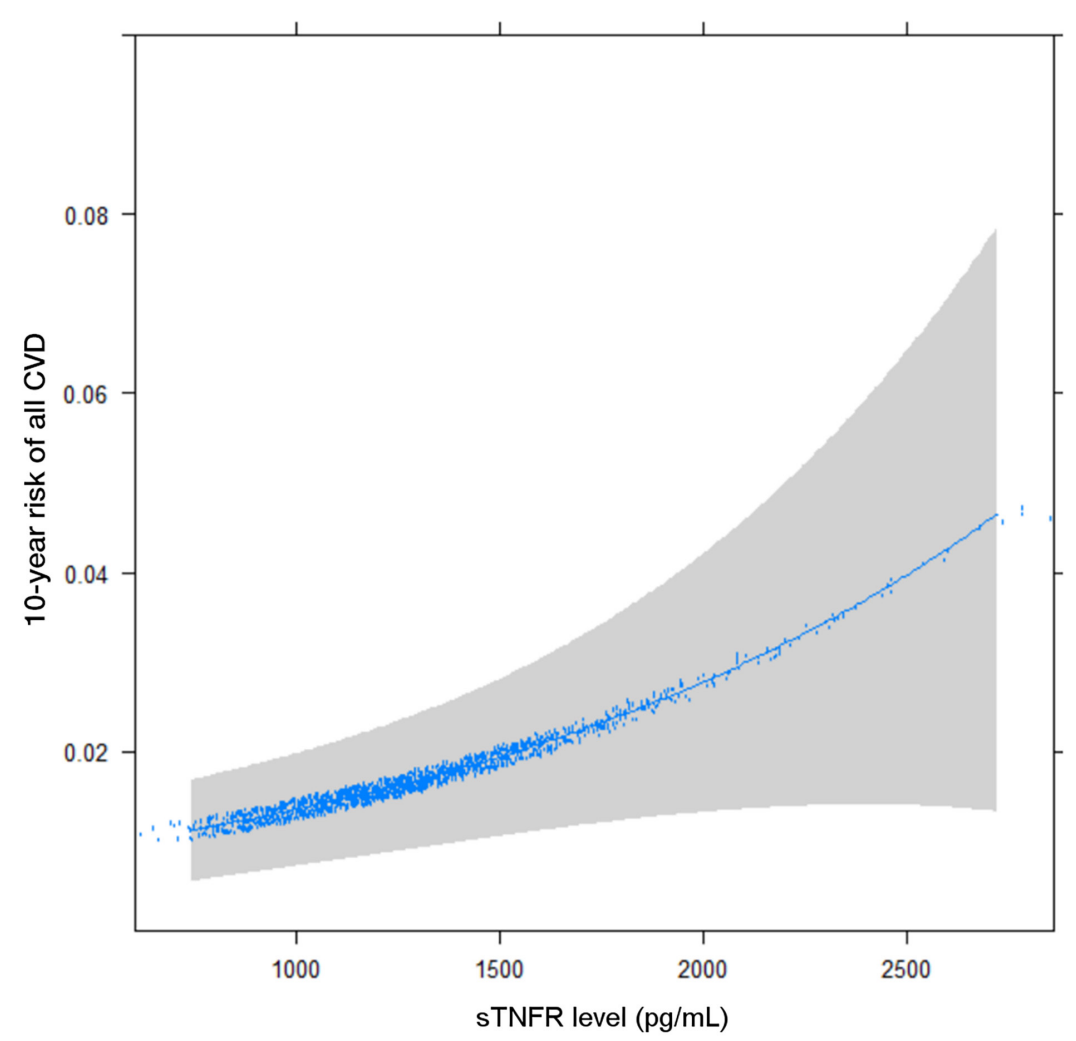

Figure 2. 10-year CVD risk by sTNFR-1 levels, adjusted for Framingham Risk Score.

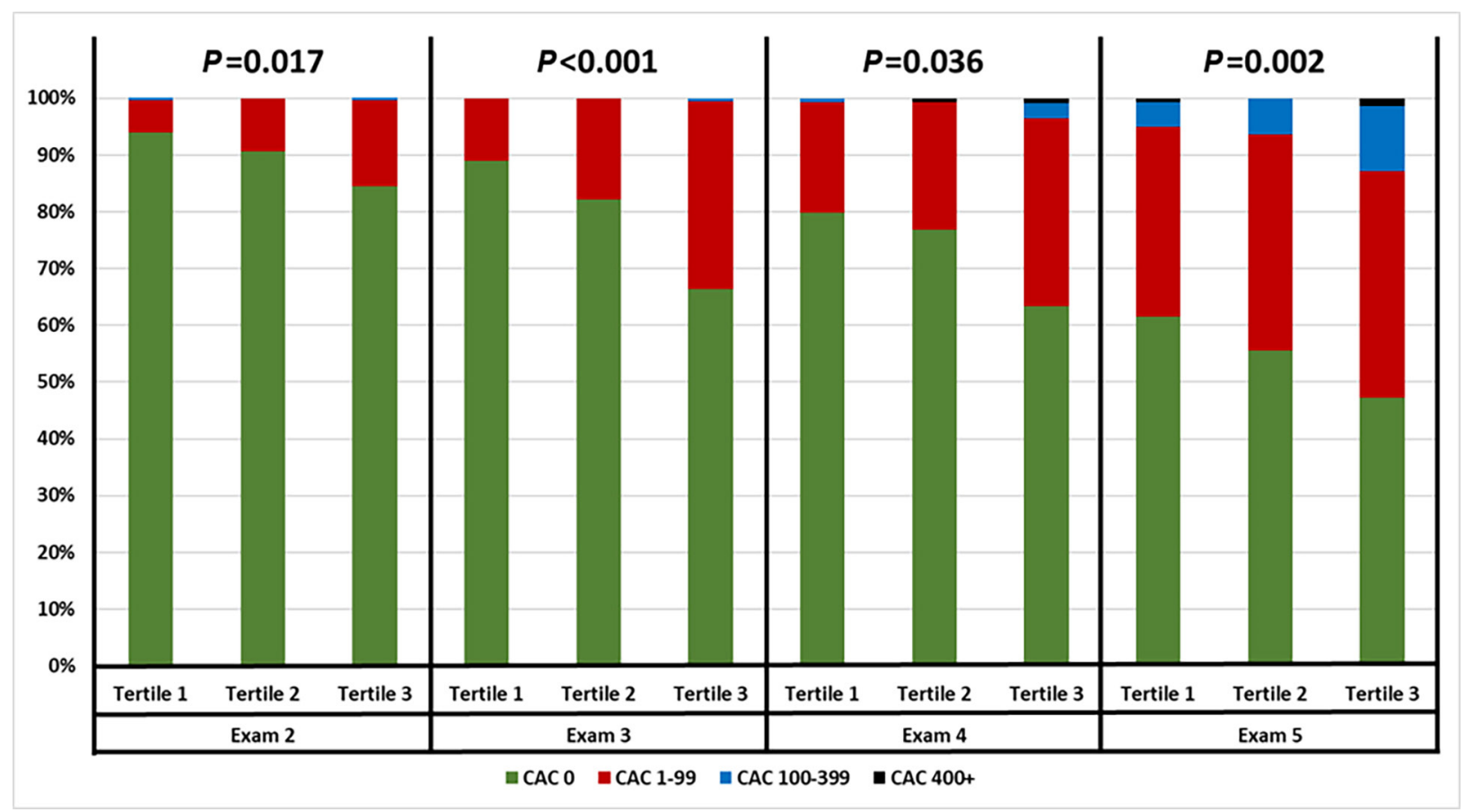

Figure 3. Coronary artery calcium score progression stratified by sTNFR-1 tertiles. 


\section{DISCUSSION}

To our knowledge, this is the first study to investigate the association between STNFR-1 and CV risk in subjects with CAC of 0 . We show that sTNFR-1 is associated with CV risk in this low-risk cohort independently of traditional CVD risk factors and improves the 10-year CVD risk stratification of the Framingham Score.

Systemic inflammation has become an increasingly recognized component in the pathogenesis of atherosclerosis and cardiovascular disease. TNF- $a$ plays a key role in the pathogenesis of systemic inflammation [18]. Mechanistically, TNF- $\alpha$ initiates its pro-inflammatory effects by binding to 2 TNF- $\alpha$ receptors TNFR- 1 and TNFR-2. These receptors are initially synthesized as membrane-anchored proteins but are subsequently released as soluble receptors that circulate in the blood (sTNFR-1 and sTNFR-2). When cellular TNF- $\alpha$ activity increases in the presence of pro-inflammatory stimuli, the level of sTNFR-1 and sTNFR-2 correspondingly increase in the blood [7]. Due to the long serum half-life of sTNFR-1, it is a measurable and stable marker of ongoing TNF cytokine activity and thus is a useful correlate to systemic inflammation.

Due to the key role of TNF- $\alpha$ in systemic inflammation, it has been a target for immunological therapies in autoimmune conditions like inflammatory bowel disease [19], rheumatoid arthritis [20], and psoriasis [21]. In coronary artery disease, TNF- $\alpha$ is expressed in atherosclerotic plaque [22] and plays a direct role in plaque destabilization [23]. Indeed, sTNFR-1 has been associated with increased arterial stiffening [24] , and elevated TNF- $\alpha$ levels have been associated with recurrent cardiovascular events in post-MI patients []]. Observational studies have also revealed a decreased incidence of MI and stroke in patients receiving anti-TNF therapies for rheumatoid arthritis compared to immunologic naive patients [25]. In those without established pro-inflammatory conditions, adipose tissue may be a driver of TNF- $\alpha$ levels [26], and some intensive diet/ exercise interventions are associated with its reduction [27]. Thus, the association between sTNFR-1 with BMI in our study is consistent with the idea that inflammatory risk may also be modified by lifestyle.

Experimental and observational studies have linked TNF signaling with the pathogenesis of atherosclerosis. TNF has been linked to endothelial injury [11]], endothelial apoptosis [28], and impairment of endothelial progenitor cells [29] , partly through reactive oxygen and nitrogen intermediates $[\underline{30}, \underline{31}]$, resulting in recruitment of inflammatory cells [32] leading to atherosclerosis progression. Small studies have shown that treatment of patients with chronic inflammatory conditions with TNF inhibitors showed short-term improvement in markers of endothelial function $[\underline{33}, \underline{34}]$. Our observation linking TNF with progression suggests that sTNFR-1 can act as a marker of residual inflammatory risk, even in patients without evidence of overt atherosclerosis, and warrants further studies for selective inhibition of TNF and traditional CV risk reduction strategies (eg, lipid-lowering therapy) in patients with elevated sTNFR-1.

A CAC score of zero is an important negative risk marker that imparts a CVD free "warranty period" of 3 to 7 years in patients without prior history of CVD [35]. In agreement with this, the 2018 ACC/AHA guidelines recommend against initiation of statins in patients with a CAC of zero as long as the 10-year ASCVD risk is less than 20\% [36]. The incidence of CVD in our study was low $(2.5 \%)$ as expected. However, the consideration of systemic inflammation may be important on an individual level even in patients with a CAC of zero as it could lead to undetected noncal- 
cified plaque [14], an increased rate of plaque formation, and risk of plaque rupture. For these patients, traditional risk factors may not fully capture the individualized 10-year CVD risk. This is further supported by the fact that sTNFR-1 improves the discriminative power of Framingham risk in this population as well as the increased rate of calcium score progression with increasing sTNFR-1 tertiles.

Our study highlights sTNFR-1 as an important inflammatory biomarker to consider in patients with a CAC of zero, as it reflects an increased risk of future CVD events. In the MESA cohort, sTNFR-1 as a predictive marker appears to be better than other commonly used inflammatory markers (interleukin-6 and CRP). In association with other traditional risk scores, it may offer added value in CVD risk stratification. It may be reasonable to initiate lipid-lowering therapies or aggressive lifestyle interventions in such patients without CAC, but with a high level of systemic inflammation.

One limitation in this study is the relatively low incidence of CVD due to the low-risk population and relatively short follow-up time, resulting in reduced statistical power. Additionally, this study does not prove causation between sTNFR-1 and CVD events. However, this analysis is strengthened by using a prospective community-based cohort with adjudicated cardiovascular events. Lastly, the upper limit of CVD risk in this patient population did not reach the $7.5 \%$ (a common cut-off used for statin initiation). This was likely due to the low-risk profile of this asymptomatic cohort and the relatively low sTNFR-1 values compared to patients with autoimmune conditions like inflammatory bowel disease [37]. Future work should investigate further the utility of adding sTNFR-1 as a biomarker for CVD risk stratification, as well as the benefit of statins in patients with systemic inflammation despite a zero CAC score.

In conclusion, sTNFR-1 concentrations are associated with an increased CVD risk in patients with a CAC of zero, independently of traditional CVD risk factors. Utilizing sTNFR-1 measurements may help to improve cardiovascular risk stratification and guide primary prevention interventions in the future. These findings may have implications for CVD prevention in patients with chronic inflammatory disease and CAC score of zero.

\section{CONFLICTS OF INTERESTS}

The authors declare that they have no known competing financial interests or personal relationships that could have appeared to influence the work reported in this paper.

\section{SUPPLEMENTARY DATA}

Supplementary materials are available at the Pathogens and Immunity website. Supplementary data may be provided by the authors to benefit the reader. Supplementary data are not copyedited and are the sole responsibility of the authors. Questions or comments related to supplementary materials should be addressed to the corresponding author.

Supplementary Tables 1 and 2

Supplementary Figure 1

\section{ACKNOWLEDGEMENTS}

This manuscript was prepared using MESA Research Materials obtained from the NHLBI Bio- 
logic Specimen and Data Repository Information Coordinating Center and does not necessarily reflect the opinions or views of MESA or the NHLBI.

\section{FUNDING SOURCES}

The authors report no funding was obtained related to this study.

\section{REFERENCES}

1. Weber C, Noels H. Atherosclerosis: current pathogenesis and therapeutic options. Nature medicine. 2011;17(11):1410-1422. doi: 10.1038/nm.2538.

2. Libby P, Ridker PM, Hansson GK. Inflammation in atherosclerosis: from pathophysiology to practice. Journal of the American College of Cardiology. 2009;54(23):21292138. doi: $\underline{10.1016 / j . j a c c .2009 .09 .009 .}$.

3. Ridker PM. C-reactive protein and the prediction of cardiovascular events among those at intermediate risk: moving an inflammatory hypothesis toward consensus. Journal of the American College of Cardiology. 2007;49(21):2129-2138. doi: 10.1016/j. jacc.2007.02.052.

4. Ridker PM, Paynter NP, Rifai N, Gaziano JM, Cook NR. C-reactive protein and parental history improve global cardiovascular risk prediction: the Reynolds Risk Score for men. Circulation. 2008;118(22):2243-2251, 4p following 2251. doi: 10.1161/CIRCULATIONAHA.108.814251.

5. Ridker PM, Danielson E, Fonseca FAH, et al. Rosuvastatin to prevent vascular events in men and women with elevated C-reactive protein. The New England Journal of Medicine. 2008;359(21):2195-2207. doi: 10.1056/NEJMoa0807646.

6. Ridker PM, Everett BM, Thuren T, et al. Antiinflammatory Therapy with Canakinumab for Atherosclerotic Disease. The New England Journal of Medicine. 2017;377(12):1119-1131. doi: 10.1056/NEJMoa1707914.

7. Xanthoulea S, Pasparakis M, Kousteni S, et al. Tumor necrosis factor (TNF) receptor shedding controls thresholds of innate immune activation that balance opposing TNF functions in infectious and inflammatory diseases. The Journal of Experimental Medicine. 2004;200(3):367-376. doi: 10.1084/jem.20040435.

8. Ridker PM, Rifai N, Pfeffer M, Sacks F, Lepage S, Braunwald E. Elevation of tumor necrosis factor-alpha and increased risk of recurrent coronary events after myocardial infarction. Circulation. 2000;101(18):2149-2153. doi: 10.1161/01.cir.101.18.2149.

9. Li XY, Hou HT, Chen HX, Wang ZQ, He GW. Increased circulating levels of tumor necrosis factor-like cytokine $1 \mathrm{~A}$ and decoy receptor 3 correlate with SYNTAX score in patients undergoing coronary surgery. The Journal of International Medical Research. 2018;46(12):5167-5175. doi: 10.1177/0300060518793787.

10. McClelland RL, Chung H, Detrano R, Post W, Kronmal RA. Distribution of coronary artery calcium by race, gender, and age: results from the Multi-Ethnic Study of Atherosclerosis (MESA). Circulation. 2006;113(1):30-37. doi: 10.1161/CIRCULATIONAHA.105.580696. 
11. Zhang C, Xu X, Potter BJ, et al. TNF-alpha contributes to endothelial dysfunction in ischemia/reperfusion injury. Arteriosclerosis, Thrombosis, and Vascular Biology. 2006;26(3):475-480. doi: 10.1161/01.ATV.0000201932.32678.7e.

12. Shareghi S, Ahmadi N, Young E, Gopal A, Liu ST, Budoff MJ. Prognostic significance of zero coronary calcium scores on cardiac computed tomography. Journal of Cardiovascular Computed Tomography. 2007;1(3):155-159. doi: 10.1016/j.jcct.2007.10.001.

13. Plank F, Friedrich G, Dichtl W, et al. The diagnostic and prognostic value of coronary CT angiography in asymptomatic high-risk patients: a cohort study. Open Heart. 2014;1(1):e000096. doi: 10.1136/openhrt-2014-000096.

14. Khan A, Arbab-Zadeh A, Kiani AN, Magder LS, Petri M. Progression of noncalcified and calcified coronary plaque by CT angiography in SLE. Rheumatology International. 2017;37(1):59-65. doi: 10.1007/s00296-016-3615-Z.

15. Bild DE, Bluemke DA, Burke GL, et al. Multi-Ethnic Study of Atherosclerosis: objectives and design. American Journal of Epidemiology. 2002;156(9):871-881. doi: 10.1093/aje/kwf113.

16. Burke G, Lima J, Wong ND, Narula J. The Multiethnic Study of Atherosclerosis. Global Heart. 2016;11(3):267-268. doi: 10.1016/j.gheart.2016.09.001.

17. Bluemke DA, Kronmal RA, Lima JAC, et al. The relationship of left ventricular mass and geometry to incident cardiovascular events: the MESA (Multi-Ethnic Study of Atherosclerosis) study. Journal of the American College of Cardiology. 2008;52(25):2148-2155. doi: 10.1016/j.jacc.2008.09.014.

18. Horiuchi T, Mitoma H, Harashima S ichi, Tsukamoto H, Shimoda T. Transmembrane TNF-alpha: structure, function and interaction with anti-TNF agents. Rheumatology (Oxford, England). 2010;49(7):1215-1228. doi: 10.1093/rheumatology/keq031.

19. Kemp R, Dunn E, Schultz M. Immunomodulators in inflammatory bowel disease: an emerging role for biologic agents. BioDrugs : Clinical Immunotherapeutics, Biopharmaceuticals and Gene Therapy. 2013;27(6):585-590. doi: 10.1007/s40259-013-0045-2.

20. Ma X, Xu S. TNF inhibitor therapy for rheumatoid arthritis. Biomedical Reports. 2013;1(2):177-184. doi: 10.3892/br.2012.42.

21. Tobin AM, Kirby B. TNF alpha inhibitors in the treatment of psoriasis and psoriatic arthritis. BioDrugs: Clinical Immunotherapeutics, Biopharmaceuticals and Gene Therapy. 2005;19(1):47-57. doi: 10.2165/00063030-200519010-00006.

22. Rus HG, Niculescu F, Vlaicu R. Tumor necrosis factor-alpha in human arterial wall with atherosclerosis. Atherosclerosis. 1991;89(2-3):247-254. doi: 10.1016/00219150(91)90066-c.

23. Wang S, Bray P, McCaffrey T, March K, Hempstead BL, Kraemer R. p75(NTR) mediates neurotrophin-induced apoptosis of vascular smooth muscle cells. The American Journal of Pathology. 2000;157(4):1247-1258. doi: 10.1016/S0002-9440(10)64640-8.

24. Kim HL, Lee JP, An JN, et al. Soluble Tumor Necrosis Factor Receptors and Arterial Stiffness in Patients With Coronary Atherosclerosis. American Journal of Hypertension. 2017;30(3):313-318. doi: 10.1093/ajh/hpw134. 
25. McKellar GE, McCarey DW, Sattar N, McInnes IB. Role for TNF in atherosclerosis? Lessons from autoimmune disease. Nature Reviews Cardiology. 2009;6(6):410-417. doi: 10.1038/nrcardio.2009.57.

26. Makki K, Froguel P, Wolowczuk I. Adipose tissue in obesity-related inflammation and insulin resistance: cells, cytokines, and chemokines. ISRN Inflammation. 2013;2013:139239. doi: 10.1155/2013/139239.

27. Albert MA, Glynn RJ, Ridker PM. Effect of physical activity on serum C-reactive protein. The American Journal of Cardiology. 2004;93(2):221-225. doi: 10.1016/j.amjcard.2003.09.046.

28. Li JH, Pober JS. The cathepsin B death pathway contributes to TNF plus IFN-gamma-mediated human endothelial injury. Journal of Immunology (Baltimore, Md : 1950). 2005;175(3):1858-1866. doi: 10.4049/jimmunol.175.3.1858.

29. Grisar J, Aletaha D, Steiner CW, et al. Endothelial progenitor cells in active rheumatoid arthritis: effects of tumour necrosis factor and glucocorticoid therapy. Annals of the Rheumatic Diseases. 2007;66(10):1284-1288. doi: 10.1136/ard.2006.066605.

30. Gao X, Zhang H, Belmadani S, et al. Role of TNF-alpha-induced reactive oxygen species in endothelial dysfunction during reperfusion injury. American Journal of Physiology Heart and Circulatory Physiology. 2008;295(6):H2242-9. doi: 10.1152/ ajpheart.00587.2008.

31. Corda S, Laplace C, Vicaut E, Duranteau J. Rapid reactive oxygen species production by mitochondria in endothelial cells exposed to tumor necrosis factor-alpha is mediated by ceramide. American Journal of Respiratory Cell and Molecular Biology. 2001;24(6):762-768. doi: 10.1165/ajrcmb.24.6.4228.

32. Quinn K, Henriques M, Parker T, Slutsky AS, Zhang H. Human neutrophil peptides: a novel potential mediator of inflammatory cardiovascular diseases. American Journal of Physiology Heart and Circulatory Physiology. 2008;295(5):H1817-24. doi: 10.1152/ ajpheart.00472.2008.

33. Spinelli FR, di Franco M, Metere A, et al. Decrease of asymmetric dimethyl arginine after anti-TNF therapy in patients with rheumatoid arthritis. Drug Development Research. 2014;75 Suppl 1:S67-9. doi: 10.1002/ddr.21200.

34. Tam LS, Kitas GD, González-Gay MA. Can suppression of inflammation by anti-TNF prevent progression of subclinical atherosclerosis in inflammatory arthritis? Rheumatology (Oxford, England). 2014;53(6):1108-1119. doi: 10.1093/rheumatology/ket454.

35. Dzaye O, Dardari ZA, Cainzos-Achirica M, et al. Warranty Period of a Calcium Score of Zero: Comprehensive Analysis From MESA. JACC Cardiovascular Imaging. 2021;14(5):990-1002. doi: 10.1016/j.jcmg.2020.06.048.

36. Greenland P, Blaha MJ, Budoff MJ, Erbel R, Watson KE. Coronary Calcium Score and Cardiovascular Risk. Journal of the American College of Cardiology. 2018;72(4):434447. doi: 10.1016/j.jacc.2018.05.027.

37. Spoettl T, Hausmann M, Klebl F, et al. Serum soluble TNF receptor I and II levels correlate with disease activity in IBD patients. Inflammatory Bowel Diseases. 
2007;13(6):727-732. doi: 10.1002/ibd.20107.

FOOTNOTES

Submitted August 10, 2021 | Accepted September 27, 2021 | Published December 3, 2021

\section{COPYRIGHT}

Copyright (c) 2021 Pathogens and Immunity. This is an open-access article distributed under the terms of the Creative Commons Attribution 4.0 International License. 